\title{
Cryopreservation of Human Adipose-Derived Stem Cells for Use in Ex Vivo Regional Gene Therapy for Bone Repair
}

\author{
Venus Vakhshori, ${ }^{1}$ Sofia Bougioukli, ${ }^{1}$ Osamu Sugiyama, ${ }^{1}$ Amy Tang, ${ }^{1}$ \\ Robert Yoho, ${ }^{2}$ and Jay R. Lieberman ${ }^{1, *}$ \\ ${ }^{1}$ Department of Orthopedic Surgery, Keck School of Medicine, University of Southern California, Los Angeles, California; \\ ${ }^{2}$ Cosmetic surgery private practice, Visalia, California.
}

The development of an ex vivo regional gene therapy clinical pathway using adipose-derived stem cells (ASCs) may require cryopreservation for cell culture, storage, and transport prior to clinical use. ASCs isolated from five donors were transduced with a lentiviral vector containing BMP-2. Three groups were assessed: transduction without cell freezing (group 1), freezing of cells for 3 weeks followed by transduction (group 2), and cell transduction prior to freezing (group 3). Nontransduced cells were used as a control. The cluster of differentiation (CD) marker profiles, cell number, BMP-2 production, and osteogenic potential were measured. The CD marker profile (CD44, CD73, CD90, and CD105) was unchanged after cryopreservation. Cell number was equivalent among cryopreservation protocols in transduced and nontransduced cells. There was a trend toward decreased BMP-2 production in group 3 compared to groups 1 and 2. Osteogenic potential based on Alizarin red concentration was higher in group 2 compared to group 3, with no difference compared to group 1. Freezing ASCs prior to transduction with a lentiviral vector containing $B M P-2$ has no detrimental effect on cell number, BMP-2 production, osteogenic potential, or immunophenotype. Transduction prior to freezing, however, may limit the BMP-2 production and potential osteogenic differentiation of the ASCs.

Keywords: cryopreservation, adipose-derived stem cells, regional gene therapy, osteogenic differentiation, BMP2

\section{INTRODUCTION}

GENE THERAPY IS A potent therapeutic approach that has shown success in treating a variety of conditions, including sickle-cell disease, macular degeneration, leukodystrophy, Wiskott-Aldrich syndrome, and lymphoid malignancies. ${ }^{1-5}$ The authors' goal is to develop an ex vivo regional gene therapy strategy to enhance bone repair in humans. This strategy has been successful in preclinical animal models of bone repair. ${ }^{6,7}$ Regional gene therapy has the potential to revolutionize the treatment of large bone defects in humans by providing a new, potent source of osteoinductive signals. In this approach, human mesenchymal stem cells (MSCs)-multipotent cells that are able to differentiate into adipose, cartilage, muscle, tendon, or bone-are transduced with a lentiviral vector containing the $B M P-2$ gene and implanted into a bony defect. ${ }^{8-10}$ MSCs may be harvested and isolated from several sources, including bonemarrow aspirates, adipose tissue, or umbilical cord blood. ${ }^{8-10}$

Compared to other cell sources, adipose-derived stem cells (ASCs) are a readily available source of MSCs, since they are harvested with minimally invasive techniques and little patient morbidity, and are able to be expanded rapidly in tissue culture. ${ }^{9,11}$ The use of ASCs has been studied for a variety of clinical therapies, including breast reconstruction, cardiac repair, and bone regeneration. ${ }^{12-15}$ Similar to other stem-cell populations, ASCs may be modified to express specific genes of interest, and therefore may be utilized in regional gene therapy applications in a clinical setting.

${ }^{*}$ Correspondence: Dr. Jay R. Lieberman, Department of Orthopedic Surgery, Keck School of Medicine at USC, 1520 San Pablo Street, Suite 2000, Los Angeles, CA 90033. E-mail: Jay.Lieberman@med.usc.edu 
However, the clinical use of autologous ASCs that have been transduced with a lentiviral vector as part of an ex vivo gene therapy approach for tissue engineering presents logistical challenges. One potential strategy would be for the lipoaspirate to be harvested, processed, expanded in tissue culture, and then transduced in the same facility where implantation will occur. Since most clinicians lack the appropriate facilities to perform cell culture and viral transduction, this strategy will require the use of a central facility to manage these activities. An option would be to ship the harvested lipoaspirate to a facility that would expand the ASCs in tissue culture, transduce the cells, and immediately return the transduced ASCs to the surgeon for reimplantation. Although this may be a reasonable strategy, there could be a significant number of cases where the patient might not be ready for a second-stage bonegrafting procedure, secondary to wound-healing issues, changes in health status, or scheduling conflicts. In this case, the cell transduction would have to be delayed. A limitation of this strategy is the finite amount of time ASCs may be maintained in culture prior to cell senescence and growth arrest. ${ }^{16}$ Therefore, ASCs may have to be stored at some point during the cell expansion and transduction process.

One method to store cells and preserve their viability is via cryopreservation. The lipoaspirate may be harvested and shipped to a facility for processing and cell expansion. In order to have the cells ready at the appropriate time for use in a patient, the expanded cells could be frozen either before or after transduction. When the patient is ready for the implantation procedure, the cells could be thawed and shipped to the clinician for use. Under proper storage conditions, cryopreserved ASCs can retain their viability and differentiation potential. ${ }^{17-20}$ However, there are no reports in the literature about cryopreservation of ASCs in the setting of regional gene therapy, wherein cells are transduced with a gene of interest prior to or after freezing.

This study assessed the cell viability, BMP-2 production, and osteogenic potential of ASCs that are transduced with a $B M P$-2-containing lentiviral vector in cells that are frozen before or after transduction in order to determine the optimal clinical protocol for cell cryopreservation prior to regional gene therapy. It was hypothesized that cryopreservation of ASCs before or after transduction would result in equivalent cell viability, protein production, and osteogenic potential compared to non-frozen cells.

\section{METHODS}

\section{Cell harvest}

Human adipose tissue was obtained as a coded, de-identified specimen from elective suctionassisted lipectomy (liposuction) procedures from the abdomen, buttock, or thigh. Cells from five donors were used (female patients aged 30, 31, 32, 38, and 42 years old). During this procedure, a tumescent solution with saline, lidocaine, and epinephrine was infused into the adipose tissue to minimize bleeding and postoperative pain. ${ }^{21}$ The raw lipoaspirate samples were then processed according to an established protocol. ${ }^{22}$ Briefly, the lipoaspirates were washed with an equal volume of Dulbecco's phosphate-buffered saline (DPBS; Caisson Laboratories, North Logan, UT) to remove debris, red blood cells, and remaining tumescent components. The washed adipose fraction was then digested with sterile type 1A $0.1 \%$ collagenase (Sigma-Aldrich, St. Louis, MO) at $37^{\circ} \mathrm{C}$ until the adipose layer no longer contained solid pieces of fat. The cells were then further processed and filtered, followed by incubation with ammonium-chloride-potassium (ACK) lysing buffer (Lonza, Allendale, $\mathrm{NJ}$ ) to remove red blood cells. After further washing with DPBS to eliminate the ACK lysis buffer, the resultant cell pellet composed of the stromal vascular fraction was re-suspended in Dulbecco's modified Eagle's medium (DMEM) $+10 \%$ fetal bovine serum (FBS) supplemented with antibiotic and antifungal agents (100 IU/mL penicillin, $100 \mu \mathrm{g} / \mathrm{mL}$ streptomycin, and $0.25 \mu \mathrm{g} / \mathrm{mL}$ amphotericin B) and plated at $5 \times 10^{6}$ cells per $10 \mathrm{~cm}$ plate.

\section{Cell culture}

The cells were counted using a TC20 ${ }^{\mathrm{TM}}$ automated cell counter (BioRad, Hercules, CA), and plated as described above in a humidified atmosphere and $5 \% \mathrm{CO}_{2}$ at $37^{\circ} \mathrm{C}$. The culture medium was changed every 3-4 days, and nonadherent cells were removed. When confluent, the adherent cells were passaged at a density of $0.8-1.0 \times 10^{6}$ cells per plate.

\section{Transduction}

Cells were transduced at passage 3 with a twostep transcriptional amplification (TSTA) system, as described in previously published protocols. ${ }^{6,23,24}$ Transduction was performed at passage 3 to avoid cell senescence and loss of osteogenic potential at higher passages. ${ }^{25}$ The TSTA system uses the GAL4-VP16 transactivator cDNA and the transgene expression vector encoding the G5 promoter and the $B M P-2$ cDNA under the control of the GAL4 responsive promoter. All lentiviral vectors 
were generated by transfecting 293T cells (American Type Culture Collection, Manassas, VA). The titers of these vectors were determined by quantifying the amount of $\mathrm{p} 24$ protein contents in vector solution by enzyme-linked immunosorbent assay (ELISA; Quantikine; R\&D Systems, Minneapolis, MN). Passage 3 cells were plated into a $10 \mathrm{~cm}$ dish for ELISA at a concentration of $1 \times 10^{6}$ cells per $5 \mathrm{~mL}$ media for ELISA or on a 24-well plate for Alizarin red staining at a concentration of $1 \times 10^{5}$ cells per $0.5 \mathrm{~mL}$ DMEM $+10 \%$ FBS with $8 \mu \mathrm{g} / \mathrm{mL}$ polybrene for Alizarin red staining. The cells were co-transduced overnight with LV-GAL4-VP16 at a multiplicity of infection (MOI) of 5, and LV-G5BMP-2 or LV-G5-GFP at a MOI of 25 at $37^{\circ} \mathrm{C}$ and $5 \% \mathrm{CO}_{2}$. These MOIs were chosen to minimize cell toxicity while maximizing BMP-2 and green fluorescent protein (GFP) expression.

\section{Cryopreservation protocol}

Each of the five adipose samples was processed, and the stromal vascular fraction was then plated and expanded in culture as described above. These ASCs of each donor were divided into three groups. Group 1 consisted of fresh ASCs that were transduced at passage 3 . Group 2 consisted of fresh ASCs that were frozen at passage 1 for 3 weeks at $-196^{\circ} \mathrm{C}$ in liquid nitrogen, followed by thawing in a $37^{\circ} \mathrm{C}$ water bath, and culture-expanded until passage 3 . Cells were subsequently transduced at passage 3 as described above. Group 3 consisted of ASCs that were transduced at passage 3 . After 2 days, these cells were frozen for 3 weeks at $-196^{\circ} \mathrm{C}$ in liquid nitrogen, followed by thawing. Each of the donors also had one sample of cells that was not transduced and treated either with or without cryopreservation to determine the effects of freezing on cell viability. Cryopreservation of ASCs was performed by placing cells into a cryovial at a concentration of $1 \times 10^{6}$ cells in $1 \mathrm{~mL}$ of a commercially available serum-free medium containing $10 \%$ dimethyl sulfoxide (DMSO; Bambanker; Wako Chemicals, Richmond, VA). The vials were then stored at $-80^{\circ} \mathrm{C}$ overnight in a Cool Cell LX freezing container (Biocision, San Rafael, CA). The next day, they were transferred to liquid nitrogen $\left(-196^{\circ} \mathrm{C}\right)$. After 3 weeks of freezing in liquid nitrogen, the cells were thawed in the cryovial using a $37^{\circ} \mathrm{C}$ water bath.

\section{Immunophenotypic characterization}

Nontransduced ASCs from three different donors had their cell surface markers analyzed prior to and after cryopreservation of nontransduced cells by fluorescence-activated cell sorting (FACS) to evaluate for the cell marker profile of MSCs as defined by the Mesenchymal and Tissue Stem Cell Committee of the International Society for Cellular Therapy. ${ }^{26}$ Passage 3 cells were prepared for FACS analysis following a standard BD Stemflow protocol. Briefly, after trypsinization, the cells were washed and resuspended in BD Pharmingen Stain Buffer (BD Biosciences, Franklin Lakes, $\mathrm{NJ}$ ) at a concentration of $5 \times 10^{6}$ cells $/ \mathrm{mL}$. A $100 \mu \mathrm{L}$ aliquot of the cell suspension was transferred to a separate tube and stained with cluster of differentiation marker (CD)73, CD90, CD105, CD44, and human MSC negative cocktail (CD34, CD11b, CD19, CCD45, and HLA-DR) antibodies. These CD markers were selected due to their important role in the characterization of MSCs, as defined by the Mesenchymal and Tissue Stem Cell Committee of the International Society for Cellular Therapy. ${ }^{26}$ After staining, the cells were incubated on ice in the dark for $30 \mathrm{~min}$. Unstained and single-color control samples were also prepared. Cells were then washed and re-suspended in $200 \mu \mathrm{L}$ of BD Pharmingen Stain Buffer. Sample analysis was performed using a BD LSR II flow cytometer (BD Biosciences), and data analysis was performed with FlowJo (FlowJo LLC, Ashland, OR).

\section{Quantification of cell number and BMP-2 production}

For the fresh ASCs (group 1) and postcryopreservation transduced ASCs (group 2), passage 3 cells were plated at $1 \times 10^{6}$ cells per dish 1 day before transduction (time point 1 ), and cell number was measured 1 week after transduction (time point 2). For the ASCs transduced prior to cryopreservation (group 3), fresh cells were harvested, expanded in culture to passage 3, transduced, and then frozen. After cryopreservation, the cells were thawed in a cryovial, plated, and cell number was quantified 1 day (time point 1) and 1 week (time point 2) after thawing.

To quantify BMP-2 production, cells were washed to remove any free lentiviral particles after the end of the overnight transduction period, then fresh media was applied, and cells were cultured for an additional $24 \mathrm{~h}$. After this incubation period, the medium of the transduced cells was harvested and analyzed using ELISA for BMP-2 quantification at two time points for each of the three groups. Each sample was run in triplicate, and the resultant values were averaged. Additionally, cell number was quantified with the $\mathrm{TC} 20^{\mathrm{TM}}$ automated cell counter. BMP-2 production was reported as nanograms per dish. 


\section{Osteogenic differentiation}

Cells were cultured in osteogenic media to induce osteogenic differentiation, with nontransduced cells serving as a control. Cells $\left(1 \times 10^{5}\right)$ were cultured with DMEM $+10 \%$ FBS, antibiotics/antifungals, $0.1 \mu \mathrm{M}$ dexamethasone, $50 \mu \mathrm{g} / \mathrm{ml}$ L-ascorbic acid, and $10 \mathrm{mM} \beta$-glycerophosphate on a 12 -well plate. Three samples per cell donor were used. Osteogenic differentiation was assessed at 7 days post transduction by Alizarin red S staining of extracellular calcium deposits. The cells were fixed with $10 \%$ formaldehyde for $10 \mathrm{~min}$, washed with PBS, and stained with Alizarin red S solution for $30 \mathrm{~min}$. Following staining, qualitative analysis was completed by imaging each well with a Revolve R4 microscope (Echo Laboratories, San Diego, CA). Quantification of Alizarin red S concentration was subsequently performed using a previously described protocol. ${ }^{27}$ Briefly, stained cells were incubated with $800 \mu \mathrm{L}$ of $10 \%$ acetic acid at room temperature for $30 \mathrm{~min}$, followed by heating to $85^{\circ} \mathrm{C}$ for $10 \mathrm{~min}$ and cooling on ice for $5 \mathrm{~min}$. The mixture was then centrifuged, and the supernatant was mixed with $200 \mu \mathrm{L}$ of ammonium hydroxide, and aliquots were transferred to a 96-well plate and read at $405 \mathrm{~nm}$ by a plate reader.

\section{Statistical analysis}

Statistical analysis was performed with IBM SPSS Statistics for Windows v22 (IBM Corp., Armonk, NY), with a significance level set at 0.05 . Wilcoxon signed-rank tests were used to compare BMP-2 production and cell number between time points. A related-samples Friedman's two-way analysis of variance test was performed to compare cell number, BMP-2 production, and Alizarin red concentration between the cryopreservation protocols. Comparisons with significant values then underwent Dunn's pairwise post hoc comparisons tests with a Bonferroni correction. Additionally, Wilcoxon signed-rank tests were used to compare immunophenotypes between fresh and thawed cells.

\section{RESULTS}

\section{Immunophenotypic characterization}

Using flow cytometry, the percentage of cells positive for MSC markers (CD73, CD90, CD105, and CD44), ${ }^{26}$ and hematopoietic stem-cell (HSC) markers (CD34, CD11b, CD19, CCD45, and HLADR) was determined before and after freezing. The ASCs expressed CD44, CD73, CD90, and CD105 with minimal expression of HSC markers. There was no significant difference in the percentage of cells positive for these markers before or after cryopreservation (Table 1).
Table 1. Percentage of cells positive for each CD marker tested before and after cryopreservation of cells, as determined by flow cytometry

\begin{tabular}{lccc}
\hline & Pre freeze $(\mathrm{n}=3)$ & \multicolumn{2}{c}{ Post freeze $(\mathrm{n}=3)$} \\
\cline { 3 - 4 } CD marker & Mean & Mean & $\mathrm{p}$-Value \\
\hline CD44 & 98.27 & 97.37 & 0.285 \\
CD73 & 98.40 & 96.27 & 0.109 \\
CD90 & 81.00 & 93.67 & 0.102 \\
CD105 & 58.33 & 48.17 & 0.285 \\
HSC & 0.70 & 0.68 & 1.000 \\
\hline
\end{tabular}

$\mathrm{CD}$, cluster of differentiation.

\section{BMP-2 production}

Both fresh and cryopreserved ASCs were successfully transduced with a lentiviral lector encoding $B M P$ - 2 . At time point 1, mean BMP-2 production in group 1 was $87.0 \pm 15.2 \mathrm{ng}$ per well, $119.9 \pm$ $50.0 \mathrm{ng}$ per well in group 2, and 89.1 $\pm 26.7 \mathrm{ng}$ per well in group $3(p=0.247)$. At time point 2 , mean BMP-2 production in group 1 was $579.8 \pm 604.0 \mathrm{ng}$ per well, $564.5 \pm 592.6 \mathrm{ng}$ per well in group 2 , and $213.1 \pm 107.9 \mathrm{ng}$ per well in group $3(p=0.074$; Fig. 1). BMP-2 production increased from time point 1 to time point 2 in both groups $1(p=0.043)$ and $3(p=0.043)$. In group 2 , there was no significant change in BMP-2 production between the two time points ( $p=0.138$; Fig. 1$)$.

\section{Cell number}

There was no difference in cell number at any time point for transduced cells in the three cryo-

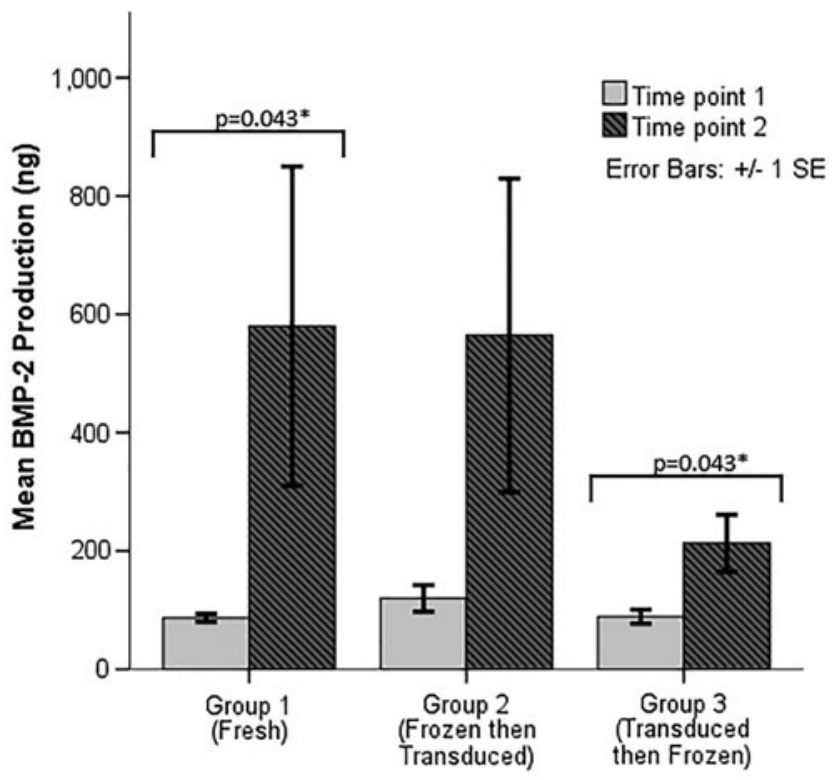

Figure 1. BMP-2 production for transduced cells. $p$-Values shown are those that are statistically significant at $<0.05$ after pairwise post hoc comparisons. 


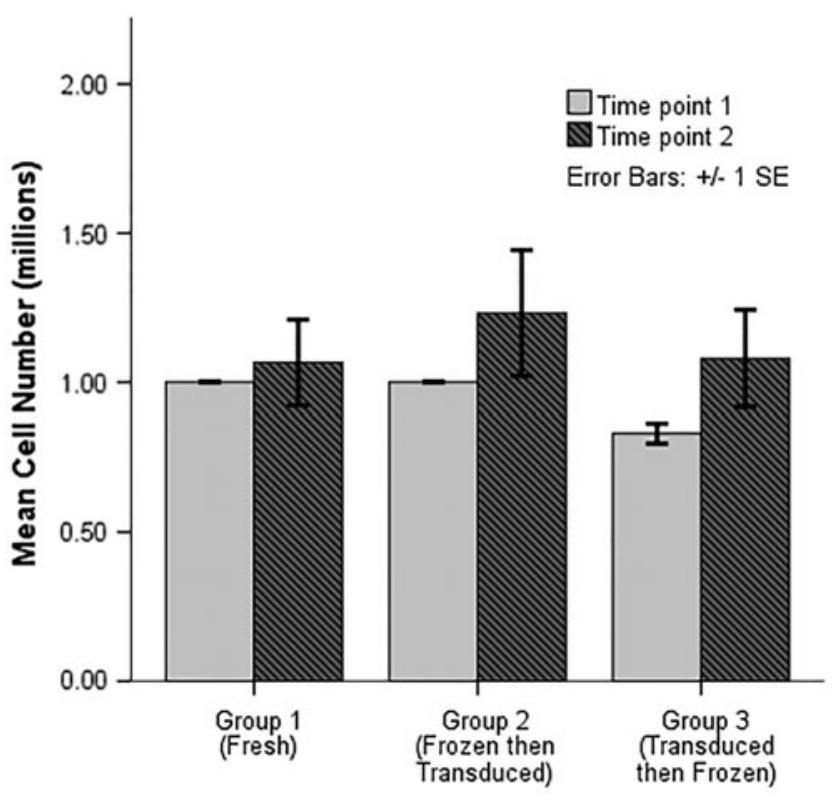

Figure 2. Cell number for transduced cells.

preservation groups. Cell number was not affected whether cells were fresh, frozen before transduction, or frozen after transduction at either time point 1 ( $p=0.053$ ) or time point 2 ( $p=0.247$; Fig. 2 ). The change in cell number between time points was not statistically significant.

In nontransduced cells, the number of cells increased at time point 2 compared to time point 1 for both fresh nontransduced $(p=0.043)$ and frozen nontransduced $(p=0.005)$ cells. Cell number was equivalent when comparing fresh and frozen cells, showing no detriment to cell viability with freezing at either time point $1(p=0.819)$ or time point 2 ( $p=0.368$; Fig. 3).

\section{Osteogenic differentiation}

Osteogenic differentiation potential, as measured at time point 2 by spectrophotometric absorbance of Alizarin red staining of extracellular calcium deposits, was significantly lower in group 3 cells compared to group 2 cells (Fig. 4). Qualitatively, there was a substantial difference in Alizarin red staining, with robust staining in transduced cells in groups 1 and 2, but minimal staining in group 3 (Fig. 5). Nontransduced cells had minimal Alizarin red staining, with no difference between fresh nontransduced and frozen nontransduced cells (Figs. 4 and 5).

\section{DISCUSSION}

ASCs are multipotent MSCs that retain the ability to differentiate into cartilage, muscle, adi-

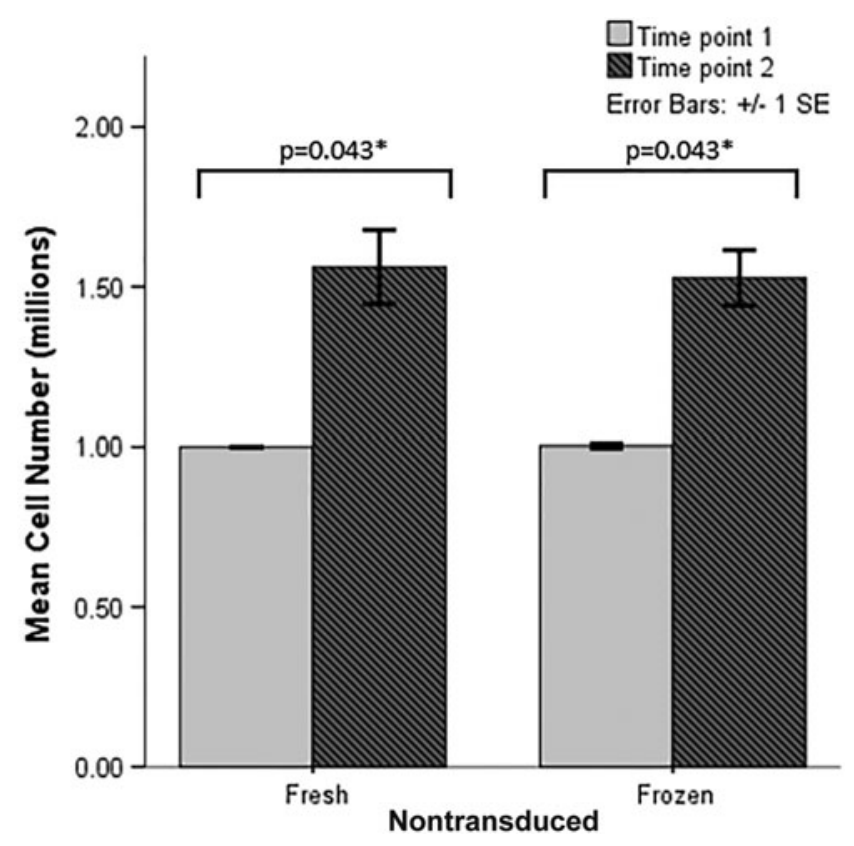

Figure 3. Cell number for nontransduced cells with and without cryopreservation. $p$-Values shown are those that are statistically significant at $<0.05$ after pairwise post hoc comparisons.

pose, and bone, making these cells prime targets for tissue engineering using regional gene therapy. ${ }^{9}$ Human adipose tissue is readily available and easily obtained from liposuction procedures. ${ }^{26} \mathrm{An}$ ex vivo regional gene therapy strategy may require expansion of cells in culture followed by transduc-

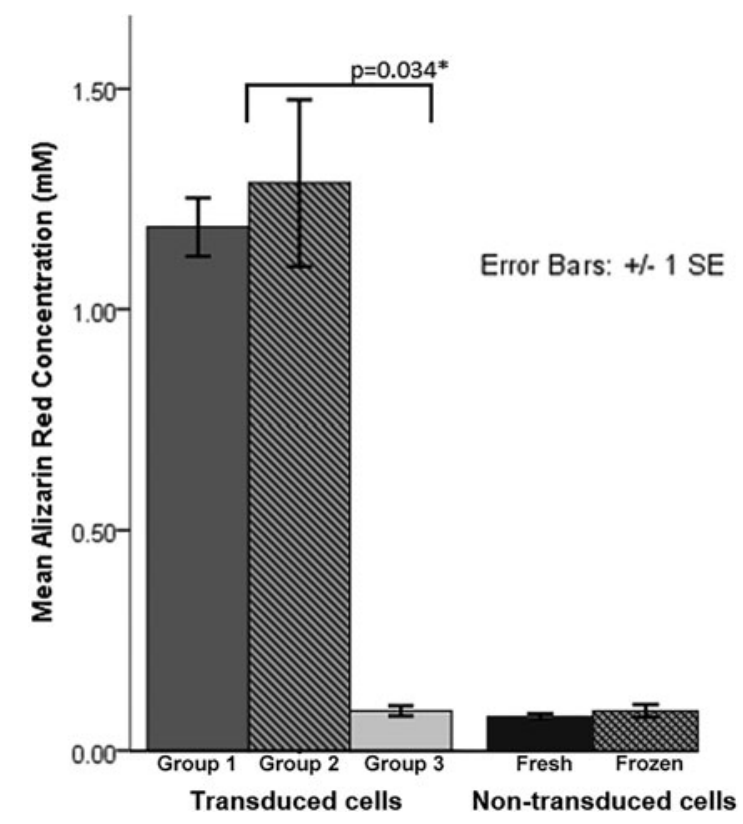

Figure 4. Alizarin red quantification for transduced cells and nontransduced cells. 


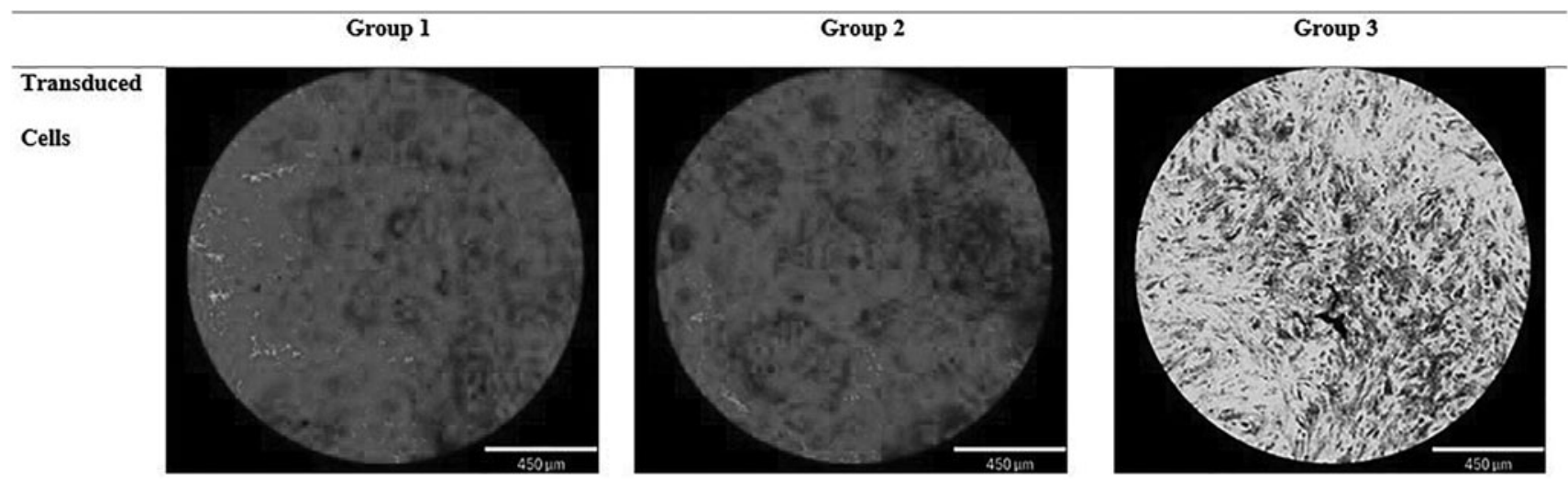

Fresh nontransduced

Frozen nontransduced

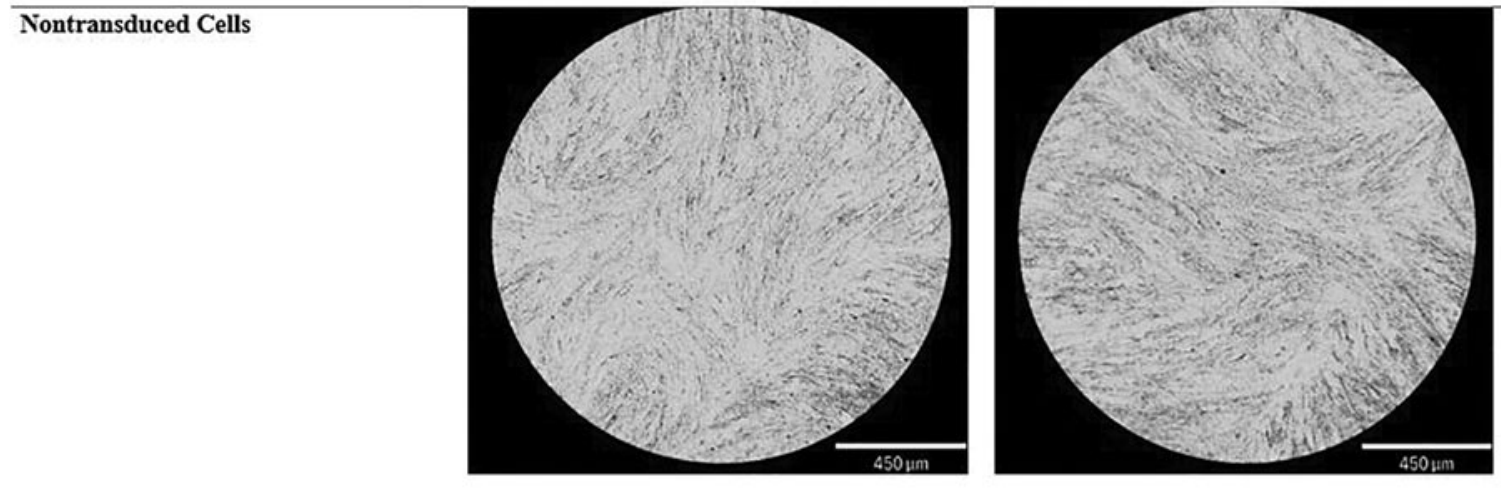

Figure 5. Representative light micrographs depicting calcium deposits detected by Alizarin red staining in the three cryopreservation protocols. Transduced cells in groups 1 (fresh transduced) and 2 (cryopreserved, then transduced) show increased extracellular calcium deposition compared to group 3 (transduced, then cryopreserved). Nontransduced fresh and nontransduced frozen cells show no extracellular calcium deposition.

tion of cultured cells with a vector carrying the gene of interest prior to reimplantation of the transduced cells at the appropriate anatomic site. This process may take days to weeks and cannot be performed at most hospitals due to the lack of highly specialized Good Manufacturing Practice (GMP) facilities. Since cell expansion and transduction will be performed at a central facility, cryopreservation allows flexibility in the timing of implantation procedures for when patients are medically optimized, by preserving cells and thus delaying the senescence and growth arrest seen for late-passage stem cells. ${ }^{28,29}$ As such, cryopreservation of cells may be a critical step in developing $e x$ vivo regional gene therapy as a clinical product.

This study demonstrates that cell number and BMP-2 production are equivalent for fresh and cryopreserved human ASCs, when cryopreservation occurs before transduction, but that there is a trend toward decreased BMP-2 production and significantly diminished differentiation into osteogenic precursors in cells transduced prior to freezing. The transduced ASCs retain their immunophenotypic characteristics after 3 weeks of cryopreservation in liquid nitrogen. The ability to produce BMP-2 in cells that were transduced after cryopreservation (group 2) was comparable to fresh transduced cells (group 1). However, BMP-2 production was lower in cells transduced prior to cryopreservation (group 3), but there was high variability in BMP-2 production between donors, and this difference was not statistically significant. The results in nontransduced cells show no difference in cell number with cryopreservation compared to non-frozen cells, suggesting that freezing alone is not a detriment to cell viability. However, transduction with a lentiviral vector containing BMP-2 followed by cryopreservation results in limited osteogenic potential, as determined by Alizarin red staining for extracellular calcium deposits. Osteogenic potential in cells transduced after cryopreservation was comparable to fresh cells. These results suggest that in the clinical adaptation of regional gene therapy, cells can be transduced after cryopreservation for storage and transport purposes without significantly impacting BMP-2 production, cell viability, or osteogenic potential. Cryopreservation after 
transduction diminishes osteogenic potential and may decrease BMP-2 production and may not be ideal for maximizing clinical benefit, but this will need to be further assessed in clinically relevant animal models.

Long-term cryopreservation is essential in reliably storing cells for clinical use. Simply freezing cells or tissue does not maintain long-term viability due to ice-crystal formation, osmotic imbalance, and membrane damage. ${ }^{30,31}$ Several studies have measured the effects of a number of variables in the cryopreservation of stem-cell populations, including rate of cooling, storage duration, and temperature in various stem-cell populations. ${ }^{32-34}$ Since rapid cooling $\left(40^{\circ} \mathrm{C} / \mathrm{min}\right)$ has a deleterious effect on cell membrane integrity, most protocols suggest cooling at a rate of approximately $1-10^{\circ} \mathrm{C} / \mathrm{min} .^{32,35-37}$ Cells may be maintained at $-80^{\circ} \mathrm{C}$ or $-196^{\circ} \mathrm{C}$. However, the majority of study protocols use liquid nitrogen storage. ${ }^{30,33,38-40}$ Gonda et al. showed that storage at $-196^{\circ} \mathrm{C}$ for 6 months does not affect the biological characteristics of ASCs. ${ }^{39}$ Cryopreservation of stem-cell populations is used to preserve cells for clinical applications as well. Detry et al. showed that after freezing peripheral blood stem cells for 3 days to 11 years at $-80^{\circ} \mathrm{C}$, followed by autologous transplantation, there was no difference in neutrophil or platelet recovery. ${ }^{33}$ Lisenko et al. showed comparable results, with controlled rate freezing at $-152^{\circ} \mathrm{C}$ for $>5$ years leading to successful hematopoietic recovery. ${ }^{34}$

Additionally, cryopreservation protocols are currently widely used in the storage of autologous chondrocytes for the treatment of focal articular cartilage defects. ${ }^{41-44}$ In this setting, cryopreservation not only allows for reliable storage of chondrocytes, but also provides patients and clinicians flexibility in scheduling implantation procedures, as well as the possibility of creating allogeneic tissue banks. ${ }^{41}$ In 2014, Mendicino et al. reviewed 66 proposals submitted to the Food and Drug Administration for MSC-based products, of which $>80 \%$ proposed cryopreservation to store and transport cells. ${ }^{42}$ In these protocols, cells are harvested, placed into a culture medium, and shipped to a commercial laboratory, where cells are expanded and frozen for storage. Once required by the clinician, the cells are thawed and shipped for implantation. ${ }^{41,42}$ Autologous chondrocyte implantation has shown long-term success in treating focal cartilage defects with no apparent detriment from cryopreservation. ${ }^{43,44}$

Despite the numerous studies evaluating cryopreservation in various stem-cell populations, few studies have assessed cryopreservation in cells that have been transduced for the purpose of gene therapy applications. Suh et al. showed that cryopreserved corneal endothelial cells retained their phenotypic properties, proliferative potential, gene expression, and ability to be transduced with a lentiviral vector containing a GFP gene. ${ }^{45}$ The present results were similar; the immunophenotype of the ASCs was not affected by cryopreservation, and the cells continued to express markers specific for MSCs. ASCs that were cryopreserved prior to transduction maintain their ability to proliferate, to be transduced and produce BMP-2, and to differentiate into osteogenic precursors. Gülen et al. showed that dendritic cells transduced with an adenovirus vector containing an interleukin-12 transgene may be cryopreserved with little effect on cell viability and transgene expression. ${ }^{46} \mathrm{Li}$ et al. showed that cord blood myeloid progenitor cells can be transduced with a retroviral vector containing a neomycin resistance gene after cryopreservation, and that transduced cells successfully recovered gene expression after cyropreservation. ${ }^{47}$ The present results differ in that ASCs transduced prior to cryopreservation had diminished osteogenic potential and a trend toward decreased BMP-2 production. The reason for this difference may be related to the use of a different cell type, viral vector, and transgene. ${ }^{46,47}$ To the authors' knowledge, this study is the first to evaluate cryopreservation in genetically modified ASCs that are transduced with a $B M P-2$ containing vector as part of a gene therapy strategy for bone repair.

This study has several limitations. First, while in vitro preservation of cell viability, osteogenic differentiation, protein production, and immunophenotype is shown after cryopreservation of ASCs, it is unclear if cryopreserved ASCs retain their function in vivo. Second, cells from five donors were tested in this study, and all five donors' cells were included in each of the freezing conditions to limit variability. Despite this, there was a large variation in the amount of BMP-2 produced between donors, leading to large standard deviations, which limits the statistical power of comparisons between groups and time points. Third, only one duration, rate, and temperature for cryopreservation were tested. ${ }^{33,34}$ Given previously published results, storage duration and temperature do not have a significant effect on stem-cell function. Therefore, multiple durations and temperatures were not tested..$^{33,34,39}$ Finally, DMSO was used as a cryoprotectant in a serum-free solution. Thirumala et al. and Yong et al. both demonstrated that postthaw ASC viability and osteogenic potential can be 
maintained in serum-free cryoprotectant solution in the presence of varying concentrations of DMSO. ${ }^{48,49}$ While DMSO is a commonly used cryoprotective agent both in vitro and clinically, increased DMSO concentrations may lead to cardiovascular and respiratory complications in humans. ${ }^{35,50-53}$

The results show that cells can be frozen in liquid nitrogen for 3 weeks without any adverse effects to cell viability, protein production, osteogenic potential, or immunophenotype. In a clinical setting, this suggests that cells can be transduced with the gene of interest-BMP-2 in the case of bone healing - at any time after cryopreservation, without significantly impacting protein production, cell viability, or osteogenic potential, provided the cells are maintained in proper storage conditions. Cryopreservation eliminates time constraints in scheduling implantation procedures. When treating a patient with a fracture nonunion or large bone defect, adipose tissue may be harvested and then shipped to a GMP facility for cell isolation, expansion in culture, and cryopreservation. Once the cell implantation procedure is scheduled, the cells can be thawed and transduced with a lentiviral vector containing $B M P-2$ prior to shipment to the clinician once the implantation procedure is scheduled. This ex vivo regional gene therapy strategy needs to be evaluated in a clinically relevant animal model to assess the osteoinductive potential of these transduced ASCs after cryopreservation.

\section{ACKNOWLEDGMENTS}

This work was supported by a research grant from the National Institute of Health to J.R.L. (R01AR057076). Special thanks to Judy Yoho who assisted in obtaining the adipose samples.

\section{AUTHOR DISCLOSURE}

All authors state no competing financial interests exist.

\section{REFERENCES}

1. Ribeil J-A, Hacein-Bey-Abina S, Payen E, et al Gene therapy in a patient with sickle cell disease. N Engl J Med 2017;376:848-855.

2. Heier JS, Kherani $S$, Desai $S$, et al. Intravitreous injection of AAV2-sFLT01 in patients with advanced neovascular age-related macular degeneration: a Phase 1, open-label trial. Lancet 2017; 390:50-61

3. Sessa M, Lorioli L, Fumagalli $F$, et al. Lentiviral haemopoietic stem-cell gene therapy in earlyonset metachromatic leukodystrophy: an ad-hoc analysis of a non-randomised, open-label, Phase 1/2 trial. Lancet 2016;388:476-487.

4. Castiello MC, Scaramuzza S, Pala F, et al. B-cell reconstitution after lentiviral vector-mediated gene therapy in patients with Wiskott-Aldrich syndrome. J Allergy Clin Immunol 2015;136:692702.e2.

5. Grupp SA, Kalos M, Barrett D, et al. Chimeric antigen receptor-modified $T$ cells for acute lymphoid leukemia. N Engl J Med 2013;368:15091518

6. Alaee F, Bartholomae C, Sugiyama 0, et al. Biodistribution of LV-TSTA transduced rat bone marrow cells used for "ex-vivo" regional gene therapy for bone repair. Curr Gene Ther 2015;15: 481-491.

7. Lieberman JR, Daluiski A, Stevenson S, et al The effect of regional gene therapy with bone morphogenetic protein-2-producing bone-marrow cells on the repair of segmental femoral defects in rats. J Bone Joint Surg Am 1999;81: 905-917.
8. Pittenger MF, Mackay AM, Beck SC, et al. Multilineage potential of adult human mesenchymal stem cells. Science 1999;284:143-147.

9. Zuk PA, Zhu M, Mizuno H, et al. Multilineage cells from human adipose tissue: implications for cellbased therapies. Tissue Eng 2001;7:211-228.

10. Smith JR, Cromer A, Weiss ML. Human umbilical cord mesenchymal stromal cell isolation, expansion, cryopreservation, and characterization. Curr Protoc Stem Cell Biol 2017;41:1F.18.1-1F.18.23.

11. Locke M, Windsor J, Dunbar PR. Human adiposederived stem cells: isolation, characterization and applications in surgery. ANZ J Surg 2009;79:235244.

12. O'Halloran N, Courtney D, Kerin MJ, et al. Adipose-derived stem cells in novel approaches to breast reconstruction: their suitability for tissue engineering and oncological safety. Breast Cancer (Auckl) 2017;11:1178223417726777.

13. Tajima S, Tobita M, Mizuno H. Current status of bone regeneration using adipose-derived stem cells. Histol Histopathol 2018;33:619-627.

14. Roura S, Gálvez-Montón C, Mirabel C, et al Mesenchymal stem cells for cardiac repair: are the actors ready for the clinical scenario? Stem Cell Res Ther 2017;8:238

15. Gimble JM, Guilak F, Bunnell BA. Clinical and preclinical translation of cell-based therapies using adipose tissue-derived cells. Stem Cell Res Ther 2010;1:19.

16. Mantovani C, Terenghi G, Magnaghi V. Senescence in adipose-derived stem cells and its im- plications in nerve regeneration. Neural Regen Res 2014;9:10-15.

17. Roato I, Alotto D, Belisario DC, et al. Adipose derived-mesenchymal stem cells viability and differentiating features for orthopaedic reparative applications: banking of adipose tissue. Stem Cells Int 2016;2016:4968724.

18. Choudhery MS, Badowski M, Muise A, et al Cryopreservation of whole adipose tissue for future use in regenerative medicine. J Surg Res 2014;187:24-35.

19. Minonzio G, Corazza M, Mariotta L, et al. Frozen adipose-derived mesenchymal stem cells maintain high capability to grow and differentiate. Cryobiology 2014;69:211-216.

20. Miyagi-Shiohira C, Kurima K, Kobayashi N, et al. Cryopreservation of adipose-derived mesenchymal stem cells. Cell Med 2015;8:3-7.

21. Chia CT, Neinstein RM, Theodorou SJ. Evidencebased medicine: liposuction. Plast Reconstr Surg 2017:139:267e-274e

22. Zhu M, Heydarkhan-Hagvall S, Hedrick M, et al Manual isolation of adipose-derived stem cells from human lipoaspirates. J Vis Exp 2013;e50585.

23. Iyer $M$, Wu L, Carey $M$, et al. Two-step transcriptional amplification as a method for imaging reporter gene expression using weak promoters. Proc Natl Acad Sci U S A 2001;98:14595-14600.

24. Bougioukli S, Sugiyama 0, Pannell W, et al. Gene therapy for bone repair using human cells: superior osteogenic potential of BMP-2 transduced mesenchymal stem cells derived from adipose 
tissue compared to bone marrow. Hum Gene Ther 2018:29:507-519.

25. Gruber HE, Somayaji S, Riley F, et al. Human adipose-derived mesenchymal stem cells: serial passaging, doubling time and cell senescence. Biotech Histochem 2012;87:303-311.

26. Dominici M, Le Blanc K, Mueller I, et al. Minimal criteria for defining multipotent mesenchymal stromal cells. The International Society for Cellular Therapy position statement. Cytotherapy 2006;8:315-317.

27. Gregory CA, Gunn WG, Peister A, et al. An Alizarin red-based assay of mineralization by adherent cells in culture: comparison with cetylpyridinium chloride extraction. Anal Biochem 2004;329:7784.

28. Moseley TA, Zhu M, Hedrick MH. Adipose-derived stem and progenitor cells as fillers in plastic and reconstructive surgery. Plast Reconstr Surg 2006; 118:121S-128S

29. Baltzer AWA, Lieberman JR. Regional gene therapy to enhance bone repair. Gene Ther 2004;11: 344-350.

30. Zanata F, Shaik S, Devireddy RV, et al. Cryopreserved adipose tissue-derived stromal/stem cells: potential for applications in clinic and therapy. Adv Exp Med Biol 2016;951:137-146.

31. Mazur P. Freezing of living cells: mechanisms and implications. Am J Physiol 1984;247:C125-142.

32. Thirumala S, Zvonic S, Floyd E, et al. Effect of various freezing parameters on the immediate post-thaw membrane integrity of adipose tissue derived adult stem cells. Biotechnol Prog 2005;21: 1511-1524.

33. Detry G, Calvet L, Straetmans N, et al. Impact of uncontrolled freezing and long-term storage of peripheral blood stem cells at $-80^{\circ} \mathrm{C}$ on haematopoietic recovery after autologous transplantation. Report from two centres. Bone Marrow Transplant 2014;49:780-785.

34. Lisenko K, Pavel P, Kriegsmann M, et al. Storage duration of autologous stem cell preparations has no impact on hematopoietic recovery after trans- plantation. Biol Blood Marrow Transplant 2017; 23:684-690.

35. Pollock K, Samsonraj RM, Dudakovic A, et al. Improved post-thaw function and epigenetic changes in mesenchymal stromal cells cryopreserved using multicomponent osmolyte solutions. Stem Cells Dev 2017:26:828-842.

36. De Rosa A, De Francesco F, Tirino V, et al. A new method for cryopreserving adipose-derived stem cells: an attractive and suitable large-scale and long-term cell banking technology. Tissue Eng Part C Methods 2009;15:659-667.

37. Devireddy RV, Thirumala S, Gimble JM. Cellular response of adipose derived passage- 4 adult stem cells to freezing stress. J Biomech Eng 2005;127: 1081-1086.

38. Lecchi L, Giovanelli S, Gagliardi B, et al. An update on methods for cryopreservation and thawing of hemopoietic stem cells. Transfus Apher Sci 2016;54:324-336.

39. Gonda K, Shigeura T, Sato T, et al. Preserved proliferative capacity and multipotency of human adiposederived stem cells after long-term cryopreservation. Plast Reconstr Surg 2008;121:401-410.

40. Shaik S, Wu X, Gimble J, et al. Effects of decade long freezing storage on adipose derived stem cells functionality. Sci Rep 2018;8:8162.

41. Coopman K, Medcalf N. From production to patient: challenges and approaches for delivering cell therapies. In: StemBook [Internet]. Cambridge, MA: Harvard Stem Cell Institute. www.ncbi.nlm.nih.gov/ books/NBK208660/ (last accessed April 16, 2018).

42. Mendicino M, Bailey AM, Wonnacott $K$, et al. MSC-based product characterization for clinical trials: an FDA perspective. Cell Stem Cell 2014;14: $141-145$.

43. Harris JD, Siston RA, Pan X, et al. Autologous chondrocyte implantation: a systematic review. J Bone Joint Surg Am 2010;92:2220-2233.

44. Pareek A, Carey JL, Reardon PJ, et al. Long-term outcomes after autologous chondrocyte implantation: a systematic review at mean follow-up of 11.4 years. Cartilage 2016;7:298-308.
45. Suh LH, Zhang C, Chuck RS, et al. Cryopreservation and lentiviral-mediated genetic modification of human primary cultured corneal endothelial cells. Invest Ophthalmol Vis Sci 2007;48:30563061.

46. Gülen D, Maas S, Julius H, et al. Cryopreservation of adenovirus-transfected dendritic cells (DCs) for clinical use. Int Immunopharmacol 2012;13:61-68.

47. Li ZH, Broxmeyer HE, Lu L. Cryopreserved cord blood myeloid progenitor cells can serve as targets for retroviral-mediated gene transduction and gene-transduced progenitors can be cryopreserved and recovered. Leukemia 1995;9:S12-16.

48. Yong KW, Pingguan-Murphy $B$, Xu F, et al. Phenotypic and functional characterization of longterm cryopreserved human adipose-derived stem cells. Sci Rep 2015;5:9596.

49. Thirumala S, Gimble JM, Devireddy RV. Evaluation of methylcellulose and dimethyl sulfoxide as the cryoprotectants in a serum-free freezing media for cryopreservation of adipose-derived adult stem cells. Stem Cells Dev 2010;19:513-522.

50. James AW, Levi B, Nelson ER, et al. Deleterious effects of freezing on osteogenic differentiation of human adipose-derived stromal cells in vitro and in vivo. Stem Cells Dev 2011;20:427-439.

51. Windrum P, Morris TCM, Drake MB, et al. Variation in dimethyl sulfoxide use in stem cell transplantation: a survey of EBMT centres. Bone Marrow Transplant 2005;36:601-603.

52. Benekli M, Anderson B, Wentling D, et al. Severe respiratory depression after dimethylsulphoxidecontaining autologous stem cell infusion in a patient with $\mathrm{AL}$ amyloidosis. Bone Marrow Transplant 2000;25:1299-1301.

53. Windrum $P$, Morris TCM. Severe neurotoxicity because of dimethyl sulphoxide following peripheral blood stem cell transplantation. Bone Marrow Transplant 2003;31:315.

Received for publication September 10, 2018; accepted after revision September 30, 2018.

Published online: October 1, 2018. 